\title{
Blue horizontal branch globular clusters towards the bulge: Terzan 9, NGC 6139 and NGC $6453^{\star}$
}

\author{
S. Ortolani ${ }^{1}$, E. Bica ${ }^{2}$, and B. Barbuy ${ }^{3}$ \\ 1 Università di Padova, Dept. di Astronomia, Vicolo dell'Osservatorio 5, I-35122 Padova, Italy and European Southern \\ Observatory, Karl Schwarzschild Strasse 2, D-85749 Garching bei München, Germany \\ 2 Universidade Federal do Rio Grande do Sul, Dept. de Astronomia, CP 15051, Porto Alegre 91500-970, Brazil \\ 3 Universidade de São Paulo, CP 3386, São Paulo 01060-970, Brazil
}

Received May 10; accepted June 7, 1999

\begin{abstract}
We present $V$ and $I$ photometry of the globular clusters Terzan 9 and NGC 6453 for the first time, and also of NGC 6139.

The three clusters are projected towards the bulge. The Colour-Magnitude Diagram morphologies reveal that the sample clusters have blue horizontal branches. This characterizes an important fraction of the clusters projected on the bulge, with implications on the early Galaxy history.

From the colour-magnitude diagrams we derive reddenings of $E(B-V)=1.95,0.77,0.70$ and distances $d_{\odot} \approx 4.9,9.4,8.5 \mathrm{kpc}$ respectively for Terzan 9 , NGC 6139 and NGC 6453 . We conclude that these metal-poor clusters are within the bulge volume.
\end{abstract}

Key words: globular clusters: Terzan 9, NGC 6139, NGC 6453 - HR diagram — stars: horizontal branch

\section{Introduction}

A major question in stellar population studies concerns the transition regions between bulge, thick disk and halo of the Galaxy. Globular clusters are tracers to investigate this question. In particular the formation scenario and extent of the bulge are not yet established (e.g. Armandroff 1989; Larson 1990; Minniti 1995; Spergel et al. 1996).

CCD data in the last decade are forming a more consistent picture of the spatial distribution and intrinsic properties of clusters in the central regions of the Galaxy.

The properties of 16 out of 17 known clusters within $5^{\circ}$ of the Galactic center were gathered and discussed in

Send offprint requests to: B. Barbuy

* Observations collected at the European Southern Observatory - ESO, Chile, proposal No. 61.E-0335.
Barbuy et al. (1998a). In that study it became clear that the inner bulge includes a non-negligible fraction of blue horizontal branch (BHB) clusters. A study in a wider region as far as $r \approx 20^{\circ}$ is necessary in order to understand the distribution of these populations of globular clusters and their membership to the halo or bulge. Minniti (1995) concluded, from density distribution models, that a disk component is not consistent for the inner parts of the globular cluster system, and he predicts the ratio of bulge/halo clusters as a function of distance. At $0.7 \mathrm{kpc}\left(r \approx 5^{\circ}\right.$ at the Galactic center) $68 \%$ of the globular clusters should belong to the halo, while at $3 \mathrm{kpc}\left(r \approx 20^{\circ}\right)$ the expected fraction is $87 \%$.

In the present paper we study Terzan $9\left(r=4.1^{\circ}\right.$ from the Galactic center), which is the last cluster within the $5^{\circ}$ region, still missing a deep CMD, as well as NGC 6139 and NGC 6453, which are, in turn, part of the sample in the surrounding $\operatorname{ring} 5^{\circ}<r<20^{\circ}$, located at $r=18.9^{\circ}$ and $5.8^{\circ}$ respectively.

As usual in the central parts of the Galaxy, two of the sample clusters, Terzan 9 and NGC 6453 have post-corecollapse structure, which is not the case of NGC 6139, however still a concentrated cluster with $c=1.80$ (Trager et al. 1995).

In Sect. 2 the observations are described. In Sects. 3, 4 and 5 the clusters Terzan 9, NGC 6139 and NGC 6453 are analysed, respectively. The concluding remarks are provided in Sect. 6.

\section{Observations and calibrations}

The three clusters were observed in the same night (July 3,1998 ) with the $1.5 \mathrm{~m}$ Danish telescope at ESO (La Silla). We employed an EFOSC camera equipped with a Loral/Lesser CCD detector C1W7 with $2052 \times 2052$ pixels. The pixel size is $15 \mu \mathrm{m}$, corresponding to 0.39 " on the sky, which provides a full field of $13^{\prime} \times 13^{\prime}$. 


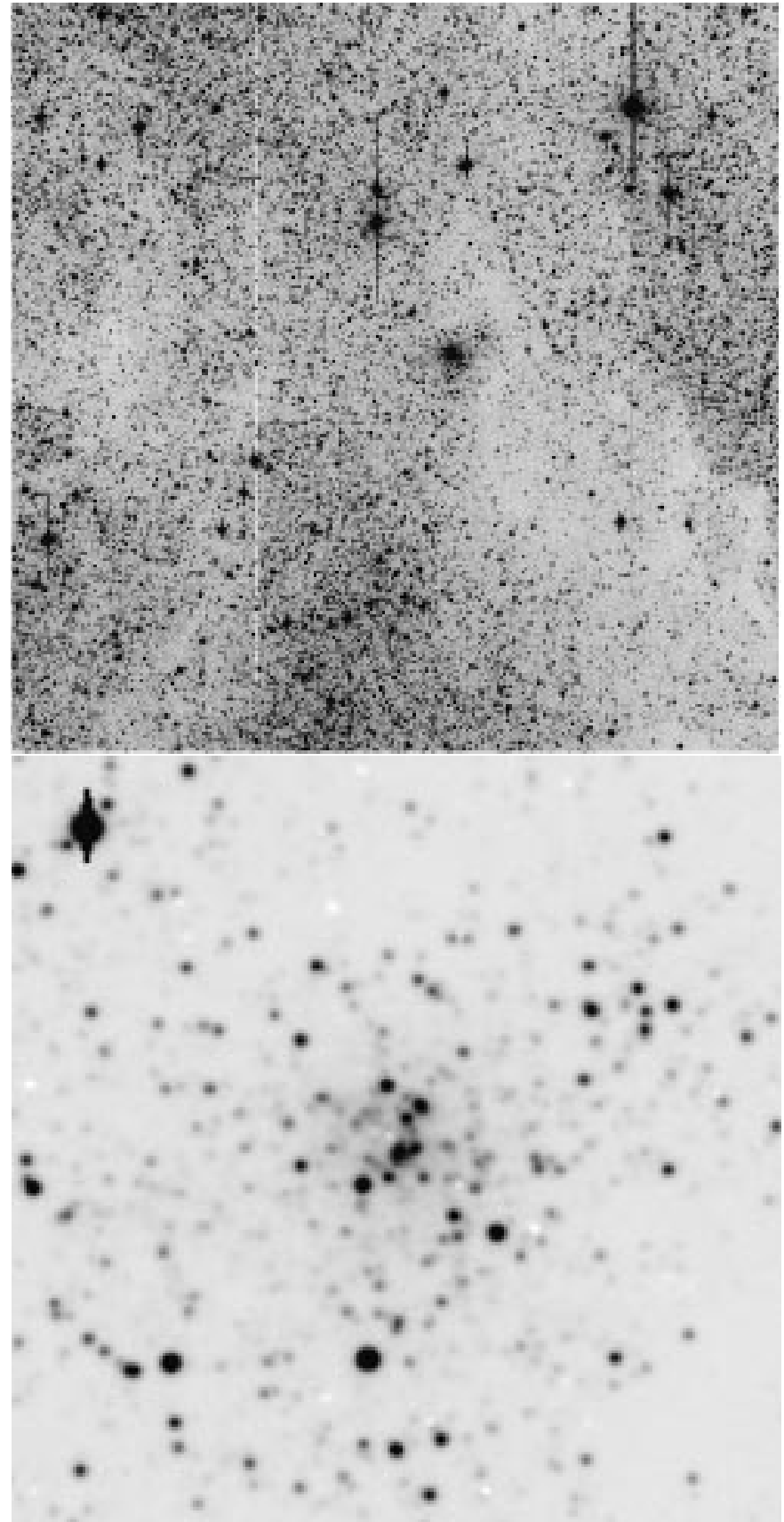

Fig. 1. $V$ image extractions of Terzan 9: a) whole field $\left(13^{\prime} \times\right.$ $\left.13^{\prime}\right)$; b) extraction of $1.6^{\prime} \times 1.6^{\prime}$ showing the cluster postcollapse core structure. North is to the top and East to the left

In Fig. 1a is shown a full field $V 15$ min exposure of Terzan 9. Notice the remarkably patchy extinction in the region. We estimated from the field stars variations of $A_{V} \approx 1.5 \mathrm{mag}$ between high and low extinction patches. In Fig. 1b we show a cluster extraction showing the core region. In Fig. 2a we show a $V$ image (3 min exposure) extraction for NGC 6139 where the populous nature of the cluster is evident. Finally, in Fig. $2 \mathrm{~b}$ we provide a $V$ extraction (1 min exposure) of NGC 6453.
Table 1. Log of observations

\begin{tabular}{llll}
\hline Target & Filter & $\begin{array}{l}\text { Exp. } \\
(\mathrm{s})\end{array}$ & $\begin{array}{l}\text { Seeing } \\
\left({ }^{\prime \prime}\right)\end{array}$ \\
\hline Terzan 9 & $V$ & 60 & 1.3 \\
& $V$ & $2 \times 900$ & 1.3 \\
& $I$ & 60 & 1.1 \\
& $I$ & $3 \times 90$ & 1.1 \\
NGC 6453 & $V$ & 60 & 1.4 \\
& $V$ & 900 & 1.4 \\
& $I$ & 10 & 1.4 \\
NGC 6139 & $I$ & 120 & 1.4 \\
& $V$ & 10 & 1.4 \\
& $V$ & 180 & 1.4 \\
& $I$ & 10 & 1.4 \\
& $I$ & 180 & 1.4 \\
\hline
\end{tabular}

The log of observations is provided in Table 1.

Daophot II was used to extract the instrumental magnitudes. For calibrations we used stars from Landolt (1983) and Landolt (1992).

Reduction procedures in such reddened crowded fields were discussed in detail in a study of Liller 1 (Ortolani et al. (1996) and references therein). The equations for the present clusters are:

$V=24.24+v$

$I=23.09+i$

reduced to $1 \mathrm{~s}$ exposure time and 1.1 airmass. Due to crowding effects arising in the transfer of the aperture magnitudes from standards to the field stars, the zero point calibration errors are dominant, estimated to be about $\pm 0.03 \mathrm{mag}$. The CCD shutter time uncertainty $(0.3 \mathrm{~s})$ for a typical $10 \mathrm{~s}$ exposure time for the standard stars, produces an additional 3\% uncertainty, which is propagated to the calibrations of the long exposure cluster frames. The final magnitude zero point uncertainty amounts to \pm 0.05 . The atmospheric extinction was corrected with the La Silla coefficients $\left(C_{V}=0.16, C_{I}=\right.$ $0.12 \mathrm{mag} /$ airmass). The zero point of the $V$ filter was checked relative to Hazen-Liller (1984) for NGC 6453, while that for NGC 6139 was done relative to Zinn \& Barnes (1998). Three bright standard stars from HazenLiller, in common to our field, give a difference $\Delta$ (Hazenpresent $)=0.015$. Three bright stars in common to Zinn $\&$ Barnes provide a difference $\Delta V($ Zinn - present $)=0.01$ \pm 0.1 and $\Delta(V-I)($ Zinn - present $)=0.01 \pm 0.2$. There is agreement between the present and previous calibrations, with no systematic trend.

\section{The globular cluster Terzan 9}

Terzan 9 is also designated GCl B1758-268 and ESO521$\mathrm{SC} 11$, and is located at $\alpha_{1950}=17^{\mathrm{h}} 58^{\mathrm{m}} 31.2^{\mathrm{s}}, \delta_{1950}=$ $-26^{\circ} 50^{\prime} 23^{\prime \prime}\left(l=3.603^{\circ}, b=-1.989^{\circ}\right)$. Malkan (1982) 


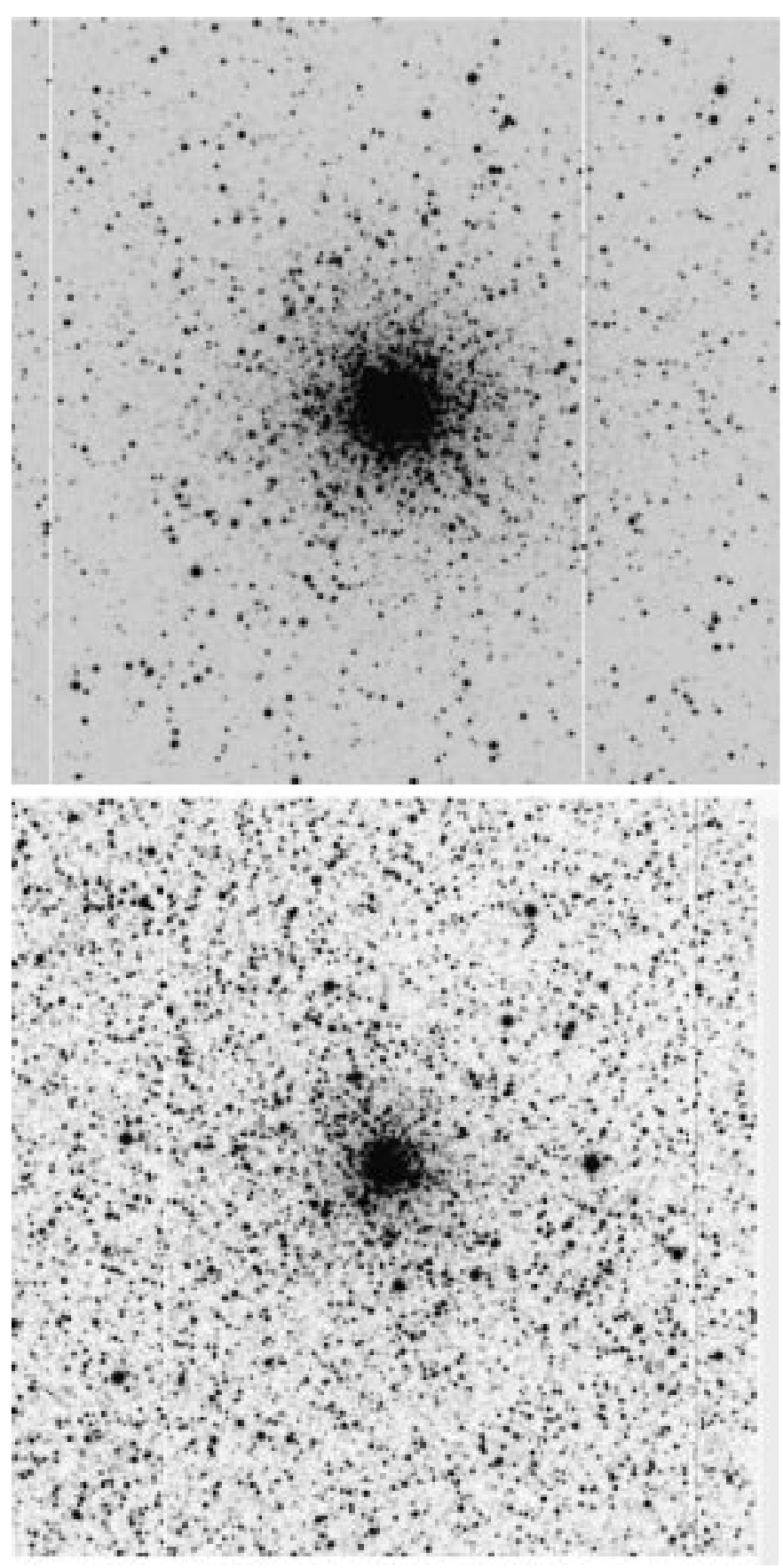

Fig. 2. $V$ image extractions of $1000 \times 1000$ pixels $\left(6.5^{\prime} \times 6.5^{\prime}\right)$ for: a) NGC 6139 ; b) NGC 6453 . Orientation as in Fig. 1

derived $E(B-V)=1.7$ from integrated infrared photometry, and Zinn (1985) based on the same data estimated a metallicity $[\mathrm{Fe} / \mathrm{H}]=-0.38$. Using near infrared integrated spectroscopy, Armandroff \& Zinn (1988) derived $[\mathrm{Fe} / \mathrm{H}]=-0.99$ from CaII triplet lines, and $E(B-V)=$ 1.25 from the interstellar band at $8621 \AA$. By means of the bright giants method Webbink (1985) estimated an horizontal branch level of $V_{\mathrm{HB}}=20.3$ which, combined to a reddening of $E(B-V)=1.71$ based on Malkan's data, led to a distance from the Sun of $d_{\odot}=7.0 \mathrm{kpc}$. Webbink
(1985) lists a metallicity $[M / H]=-0.45$ for the cluster. Liu et al. (1994) reported an infrared Colour-Magnitude Diagram $(\mathrm{CMD})$ and derived $E(B-V)=1.8$, a metallicity $[\mathrm{Fe} / \mathrm{H}]=-1.0$ and a distance from the Sun $d_{\odot}=6.9 \mathrm{kpc}$. More recently, by means of near-infrared integrated spectroscopy, Bica et al. (1998) derived $E(B-V)=1.60$ and $[\mathrm{Fe} / \mathrm{H}]=-1.01$, and pointed out that Terzan 9 was one of the clusters requiring deep photometry for more conclusive results, since bulge contamination might be occurring in the integrated light.

\subsection{Colour-magnitude diagrams}

Figure 3 a shows the $V$ vs. $(V-I)$ CMD for a $400 \times$ 400 pixel $\left(2.6^{\prime} \times 2.6^{\prime}\right)$ extraction centered on the cluster. The main features are a blue disk main sequence (MS), and a red sequence corresponding to the cluster plus bulge giants.

The best extraction for Terzan 9 is shown in Fig. $3 \mathrm{~b}$ corresponding to a radial extraction of 85 pixels $\left(33^{\prime \prime}\right)$ centered on the cluster. A vertical giant branch and a blue HB are present. This CMD is very similar to that of Terzan 4 (Ortolani et al. 1997a), an equally central cluster in the Galaxy with blue HB. Overimposed on the Terzan 9 CMD is the mean locus of M $30([\mathrm{Fe} / \mathrm{H}]=-2.13$, Zinn 1985). The mean locus of NGC $6752([\mathrm{Fe} / \mathrm{H}]=-1.54$, Zinn 1985) could be considered an acceptable fit, but not as good as with M 30. The mean loci of M 30 and NGC 6752 are from Rosino et al. (1997). Like in the case of Terzan 4, Terzan 9 is probably as metal-poor as M 30. Previous integrated methods where metallicities of $[\mathrm{Fe} / \mathrm{H}] \approx-1.0$ or higher were obtained, were certainly contaminated by metal-rich bulge field stars.

We show in Fig. 4 a $500 \times 500$ pixel extraction $\left(3.3^{\prime} \times\right.$ $\left.3.3^{\prime}\right)$ corresponding to a field CMD located at $\sim 4^{\prime}$ east of the cluster (see Fig. 1a). Besides the blue disk MS, the red $\mathrm{HB}$ of the bulge population is clearly seen at $V \approx 20.5$ and $(V-I) \approx 3.45$. The cluster RGB seen in Fig. 3a corresponds to an underpopulated zone in Fig. 4. On the other hand the bulge late type giants are seen as a curved RGB extending to very red colours, similar to metal-rich globular clusters such as NGC 6553 and NGC 6528, as well as the Baade Window (Ortolani et al. 1995 and references therein). This extension is less clear in the cluster field (Fig. 3a) because of a smaller area, crowding in the cluster area, and possibly a higher extinction.

\subsection{Reddening and distance}

The brightest part of the blue HB of Terzan 9 is located at $V_{\mathrm{HB}}=20.35 \pm 0.15$.

We calculate the cluster reddening taking M 30 as reference. By matching the overall sequences (Fig. 3b) we obtain $\Delta(V-I)=2.49$. Adopting $E(V-I) / E(B-V)=1.32$ 

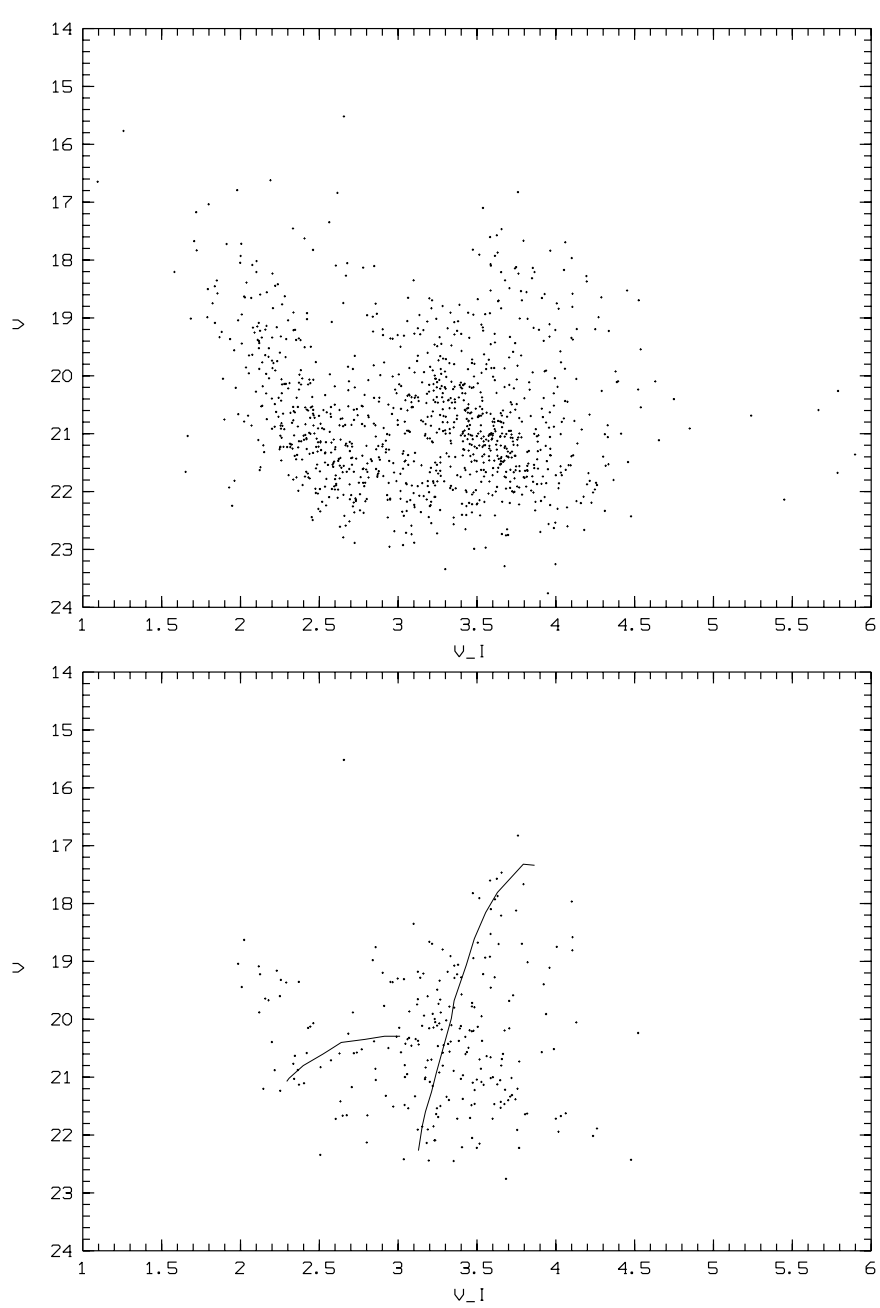

Fig. 3. $V$ vs. $(V-I)$ CMD for Terzan 9: a) $400 \times 400$ pixel extraction $\left(2.6^{\prime} \times 2.6^{\prime}\right)$ centered on the cluster, which is very contaminated by the field. b) extraction of $r<85$ pixel (1.4 ${ }^{\prime}$ radius) showing the cluster sequences. Overimposed is the mean locus of M 30

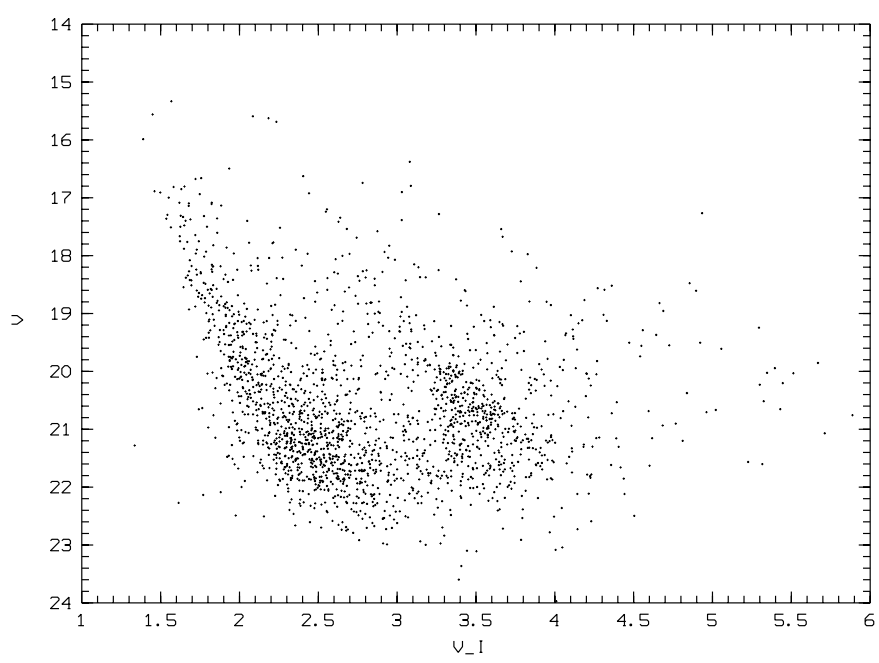

Fig. 4. $V$ vs. $(V-I)$ CMD for offset field ( $4^{\prime}$ east of Terzan 9$)$, corresponding to a $500 \times 500$ pixel area $\left(3.3^{\prime} \times 3.3^{\prime}\right)$
(Dean et al. 1978), this corresponds to $\Delta(B-V)=1.89$, and assuming $E(B-V)=0.06$ for M 30 (Barbuy et al. $1998 \mathrm{a}$ and references therein), we get $E(B-V)=1.95$, which corresponds to $A_{V}=6.24\left(R=A_{V} / E(B-V)=\right.$ 3.2 ).

Adopting an absolute magnitude of the HB for Terzan 9 like that of $\mathrm{M} 30, M_{V}=0.66$ (Barbuy et al. 1998a and references therein), we get the absolute distance modulus $(m-M)_{0}=13.45 \pm 0.2$. This corresponds to a distance of $d_{\odot}=4.9 \pm 0.7 \mathrm{kpc}$ for Terzan 9 .

To derive the Galactocentric coordinates of the cluster, we assume a distance of the Sun to the Galactic center of $R_{\odot}=8.0 \mathrm{kpc}$ (Reid 1993), to be consistent with Barbuy et al. (1998a) - notice however that Reid (1998), taking into account Hipparcos data, pointed out that $R_{\odot}$ could be as large as $9.3 \mathrm{kpc}$. Terzan 9 galactocentric coordinates are $X=-3.11$ ( $X<0$ refers to our side of the Galaxy), $Y=0.31 \mathrm{kpc}$ and $Z=-0.17 \mathrm{kpc}$. The distance to the Galactic center is $3.13 \mathrm{kpc}$, wherefrom we conclude that Terzan 9 is located within the bulge.

\subsection{Bulge field parameters}

We can calculate parameters for the offset field metal-rich bulge population and compare it with those of the metalpoor cluster. The bulge red HB is located at $V \approx 20.8$ and $(V-I) \approx 3.55$. Using the metal-rich template cluster NGC 6553 as reference (Guarnieri et al. 1998) we get $\Delta(V-I)=1.4$, corresponding to $\Delta(B-V)=1.06$. Assuming $E(B-V)=0.73$ for NGC 6553 (Barbuy et al. 1998a) we derive $E(B-V)=1.79$ for the field of Terzan 9 , which corresponds to $A_{V}=5.73(R=3.2)$. For this low Galactic latitude field $\left(l=3.6^{\circ}, b=-2.0^{\circ}\right)$ the extinction is much higher than that of the Baade Window (Terndrup 1988). Assuming $M_{V}=0.95$ for a metal-rich HB (Barbuy et al. 1998a), the distance of the bulk of the population for this bulge field results to be $7.0 \mathrm{kpc}$. However as pointed out by Barbuy et al. (1998a and references therein) the total-to-selective absorption ratio should increase with the metallicity and a more appropriate value could be $R=3.47$ for this high metallicity field, resulting in a distance of $d_{\odot}=5.1 \mathrm{kpc}$. Considering that the distance to the Galactic center is of about $8-9 \mathrm{kpc}$ (Reid $1993,1998)$, the possible interpretation is that we are observing a relatively less reddened, foreground part of the bulge, where Terzan 9 would also be embedded (Sect. 3.2).

\section{The globular cluster NGC 6139}

NGC 6139 (GCl B1624-387, ESO 331-SC4) is located at $\alpha_{1950}=16^{\mathrm{h}} 24^{\mathrm{m}} 17.4^{\mathrm{s}}, \delta_{1950}=-38^{\circ} 44^{\prime} 16^{\prime \prime}\left(l=342.37^{\circ}\right.$, $\left.b=6.94^{\circ}\right)$. The integrated Q39 photometry of Zinn (1980) provided $E(B-V)=0.78$ and $[\mathrm{Fe} / \mathrm{H}]=-1.67$, and the integrated DDO photometry of Bica \& Pastoriza (1983) 
gave $E(B-V)=0.68$ and $[\mathrm{Fe} / \mathrm{H}]=-1.28$. An integrated visible spectrum of NGC 6139 was presented by Bica \& Alloin (1986) and the line strengths were consistent with a metallicity $[\mathrm{Fe} / \mathrm{H}]=-1.5$ and the continuum distribution with $E(B-V)=0.70$. A photographic B, V CMD of NGC 6139 by Samus et al. (1996) provided $E(B-V)=0.87$ and they estimated a lower metallicity $[\mathrm{Fe} / \mathrm{H}]=-2.0$. Hazen (1991) studied the RR Lyrae in the cluster and derived $V_{\mathrm{HB}}=17.8$, and by Oosterhof group classification she argued that the cluster could be very metal-poor. Recently Zinn \& Barnes (1998) studied this cluster by means of CCD $V, I$ photometry and derived $E(B-V)=0.76,[\mathrm{Fe} / \mathrm{H}]=-1.71$ and a distance $d_{\odot} \approx 10 \mathrm{kpc}$. In the present study we compare our CMD data with those of Zinn \& Barnes (1998) and we derive the parameters with somewhat different methods, in a way consistent with our previous studies.

\subsection{Colour-magnitude diagrams}

We show in Fig. 5a the $V, I$ CMD for a $1000 \times 1000$ pixel extraction $\left(6.5^{\prime} \times 6.5^{\prime}\right)$ centered on NGC 6139 . The cluster sequences are dominant, with a blue HB and a steep RGB. The spread arises from differential reddening and some crowding effects. The blue disk MS is also present. In Fig. 5b we show a more central extraction $(250 \times 250$ pixels, or $\left.1.6^{\prime} \times 1.6^{\prime}\right)$ where the cluster sequences are welldefined, because differential reddening and field contamination are minimised. In the latter CMD only uncrowded stars are given. This CMD is suitable for measuring the cluster properties (Sect. 4.2).

Likewise Terzan 9 (Sect. 3) the best template for the giant branch distribution is that of $\mathrm{M} 30([\mathrm{Fe} / \mathrm{H}]=-2.13)$, although NGC $6752([\mathrm{Fe} / \mathrm{H}]=-1.54)$ cannot be ruled out within uncertainties. A compromise is $[\mathrm{Fe} / \mathrm{H}]=-1.80$, in agreement with the low metallicity trends found in recent stellar photometric studies (Hazen 1991; Samus et al. 1996; Zinn \& Barnes 1998). Previous integrated studies of this cluster probably included some contamination by metal-rich bulge stars.

\subsection{Reddening and distance}

The brighter parts of the blue HB of NGC 6139 are located at $V=17.98 \pm 0.10$. By taking M 30 as reference, we derive $\Delta(V-I)=1.00$, corresponding to $\Delta(B-V)=0.76$. Assuming $E(B-V)=0.06$ for M 30 we get $E(B-V)=0.82$ for NGC 6139 , which corresponds to $A_{V}=2.62(R=3.2)$.

Adopting for NGC $6139 M_{V}=0.66$, suitable for the cluster metallicity, we get the absolute distance modulus $(m-M)_{0}=14.7 \pm 0.15$. This corresponds to a distance of $d_{\odot}=8.7 \pm 0.6 \mathrm{kpc}$ for NGC 6139 .

Assuming instead NGC 6752 as template one would get $E(B-V)=0.70$ and a distance of $d_{\odot}=10.4 \pm 0.6 \mathrm{kpc}$.
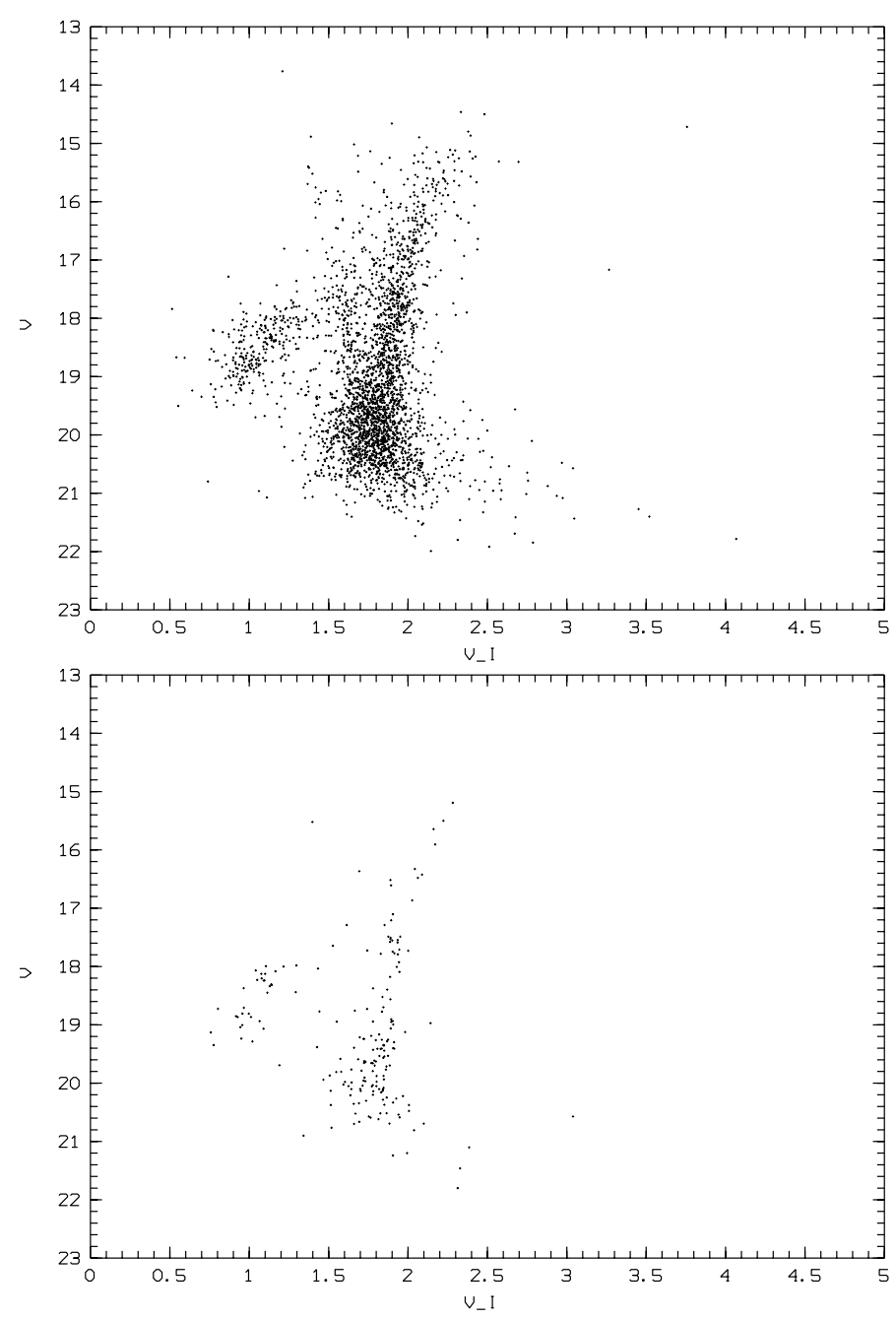

Fig. 5. $V$ vs. $(V-I)$ CMDs for NGC 6139: a) $1000 \times 1000$ pixel extraction $\left(6.5^{\prime} \times 6.5^{\prime}\right)$; b) $250 \times 250$ pixel extraction $\left(1.6^{\prime} \times 1.6^{\prime}\right)$ with selection for uncrowded stars

Giving more weight to the M 30 comparison, we adopt $E(B-V)=0.77 \pm 0.06$ and $d_{\odot}=9.4 \pm 1 \mathrm{kpc}$ for NGC 6139 . These values are comparable to those derived by Zinn \& Barnes (1998).

Assuming a distance of the Sun to the Galactic center of $R_{\odot}=8.0 \mathrm{kpc}$ as above, we get $X=0.89, Y=-2.85$ and $Z=1.14$. The cluster would be located at $R_{\mathrm{GC}}=$ $3.2 \mathrm{kpc}$ from the Galactic center.

\section{The globular cluster NGC 6453}

NGC 6453 (GCl B1747-345, ESO 393-SC36) is located at $\alpha_{1950}=17^{\mathrm{h}} 47^{\mathrm{m}} 32.0^{\mathrm{s}}, \delta_{1950}=-34^{\circ} 35^{\prime} 08^{\prime \prime}(l=$ $\left.355.72^{\circ}, b=-3.87^{\circ}\right)$. Bica \& Pastoriza (1983) derived $E(B-V)=0.60$ and $[\mathrm{Fe} / \mathrm{H}]=-1.28$. The spectroscopy of Zinn \& West (1984) gave $E(B-V)=0.60$ and $[\mathrm{Fe} / \mathrm{H}]=$ -1.53 . The visible and near-infrared spectra of Bica \& Alloin $(1986,1987)$ were consistent with $[\mathrm{Fe} / \mathrm{H}]=-1.4$ and $E(B-V)=0.61$. For this little studied cluster the 


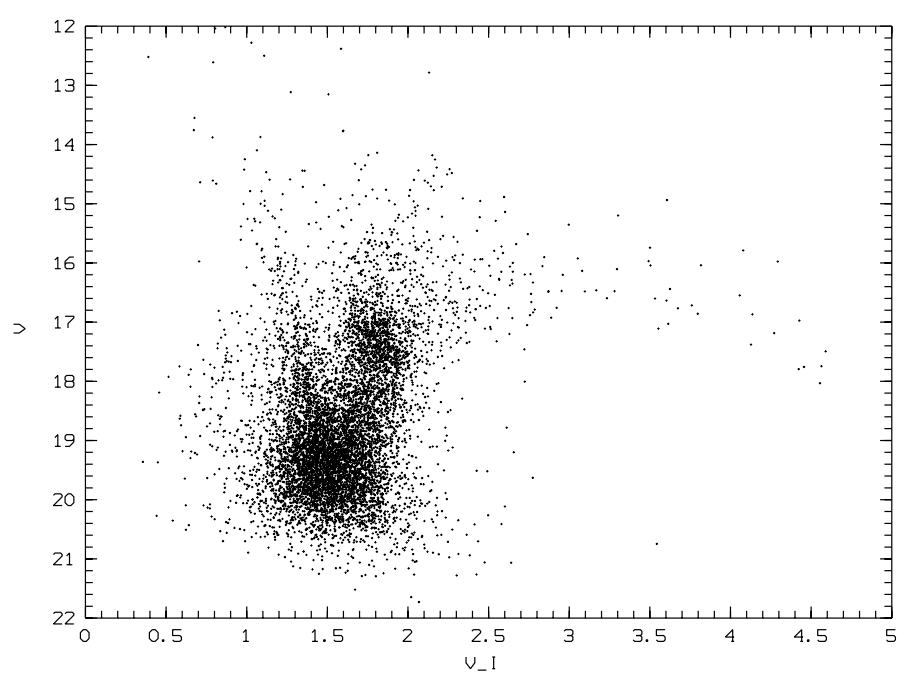

Fig. 6. $V$ vs. $(V-I)$ CMD of NGC 6453 plus field for a $1000 \times 1000$ pixel extraction $\left(6.5^{\prime} \times 6.5^{\prime}\right)$

compilations of Webbink (1985) and Harris (1996) list $E(B-V)=0.61,[\mathrm{Fe} / \mathrm{H}]=-1.52, V_{\mathrm{HB}}=17.7$ and $d_{\odot}=10.6 \mathrm{kpc}$. No CMD is available for this cluster in the literature.

\subsection{Colour-magnitude diagrams}

In Fig. 6 we give the $V, I$ CMD for a $1000 \times 1000$ pixel extraction $\left(6.5^{\prime} \times 6.5^{\prime}\right)$ centered on NGC 6453. The diagram is dominated by the metal-rich bulge population with a prominent red HB and a curved giant branch extending to very red colours, similarly to the field of Terzan 9 (Fig. 4), as well as the metal-rich globular clusters NGC 6553/NGC 6528 and the Baade Window (Ortolani et al. 1995). The blue disk MS is also important in the field of NGC 6453. The steep sequence of giants above the red HB corresponds to NGC 6453 .

In order to isolate the cluster sequences we extracted a small circular area $\left(r=150\right.$ pixels or $\left.1^{\prime}\right)$, for which the $V$ vs. $(V-I)$ CMD is given in Fig. 7. The spread can be explained by differential reddening and crowding. Nevertheless a blue HB and a steep RGB are clearly seen. Overimposed is the mean locus of M 30, suggesting a low metallicity $[\mathrm{Fe} / \mathrm{H}] \approx-2.0$, although likewise in the case of NGC 6139 (Sect. 4), a fit with the somewhat more metalrich template NGC 6752 cannot be ruled out within errors. Again, as a compromise, we adopt $[\mathrm{Fe} / \mathrm{H}]=-1.8$ for NGC 6453. This value is again somewhat lower than those from integrated studies (Sect. 1) for this cluster, which might be accounted for by some metal-rich bulge field contamination.

\subsection{Reddening and distance}

The brighter parts of the blue HB of NGC 6453 are found at $V=17.53 \pm 0.15$. Adopting M 30 as template, we get

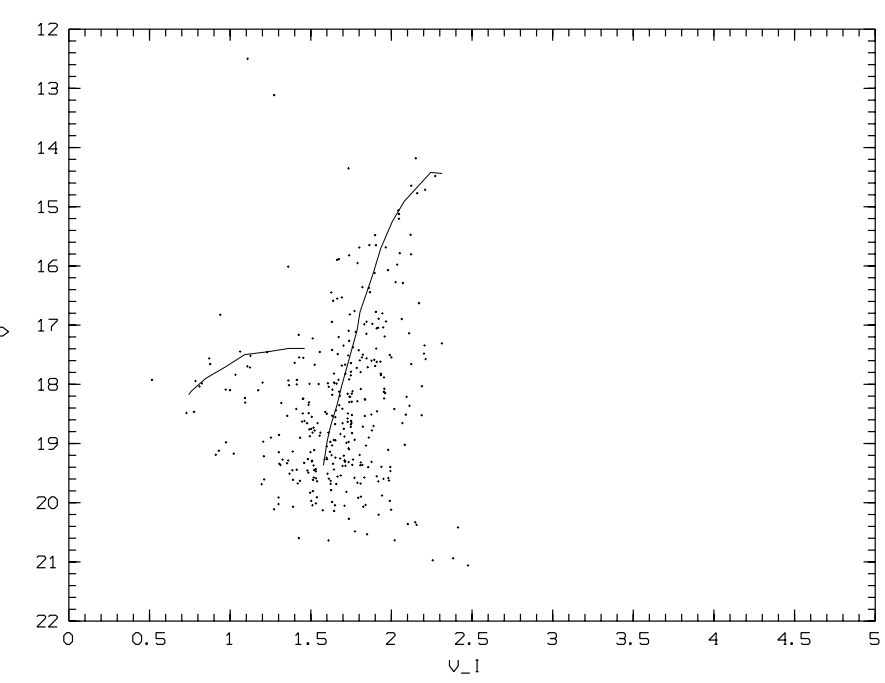

Fig. 7. $V$ vs. $(V-I)$ CMD of NGC 6453 for a radial extraction of $r=150$ pixels $\left(1^{\prime}\right)$. Overimposed is the mean locus of M 30

$\Delta(V-I)=0.90$, corresponding to $\Delta(B-V)=0.68$. With the same assumptions of Sect. 4.2, $E(B-V)=0.74$ for NGC 6453 and $A_{V}=2.37(R=3.2)$.

Adopting $M_{V}=0.66$ for the HB level as above, we get the absolute distance modulus $(m-M)_{0}=14.5 \pm 0.20$. This corresponds to a distance of $d_{\odot}=7.9 \pm 0.8 \mathrm{kpc}$ for NGC 6453. Alternatively, by adopting NGC 6752 as template one would get $E(B-V)=0.63$ and a distance of $d_{\odot}=9.4 \pm 0.6 \mathrm{kpc}$. As a compromise we adopt $E(B-V)=0.70 \pm 0.06$ and $d_{\odot}=8.5 \pm 1 \mathrm{kpc}$ for NGC 6453 .

Assuming $R_{\odot}=8.0 \mathrm{kpc}$ we derive $X=0.46, Y=$ -0.63 and $Z=-0.57$. NGC 6453 would be located at $R_{\mathrm{GC}}=1 \mathrm{kpc}$ from the Galactic center.

\subsection{Parameters for the bulge field}

Following Sect. 3.3, we calculate reddening and distance for the surrounding field of NGC 6453.

The bulge Red Horizontal Branch (RHB) is located at $V \approx 17.25$ and $(V-I) \approx 0.6$.

The template NGC 6553 is more reddened than this field under study. We derive $E(B-V)=0.46$ for the field of NGC 6453 , which gives $A_{\mathrm{V}}=1.47(R=3.2)$. The bulge extinction in this direction $\left(l=-4.3^{\circ}, b=-3.9^{\circ}\right)$ is comparable to that of the Baade Window (Terndrup 1988), which is at a similar Galactic latitude. The distance of the bulk of the population for this bulge field results to be at $9.2 \mathrm{kpc}$. Adopting $R=3.47$, it would result $d_{\odot}=8.7 \mathrm{kpc}$. This value is consistent with the Galactic center distance estimates (Reid 1993, 1998).

Finally, we note that distances for metal-poor and metal-rich globular clusters towards the bulge, and the bulk of the field population are consistent with the picture of a common volume near the Galactic Center. 


\section{Concluding remarks}

We point out that in the bulge regions we are dealing with very reddened globular clusters, contaminated by rich bulge/disk fields. The contamination affects more integrated studies than CMDs. On the other hand, since stars of different temperatures are affected differentially by effective passband variations due to reddening (Grebel \& Roberts 1995), CMD distortions are expected. We estimate that the trend will be in the sense of causing steeper RGBs. In order to get a conclusive result on the metallicity, it is required that spectroscopy of individual giants of these clusters be carried out with large telescopes.

Terzan 9, NGC 6139 and NGC 6453 are spatially located within the bulge volume. Since they appear to be metal-poor, it is not clear whether they belong to the bulge or halo population. Kinematical data would help constrain this issue.

In addition to the results for Terzan 9, NGC 6139 and NGC 6453 , a series of recent CCD photometries of clusters towards the bulge showed that an important sample has blue horizontal branches, namely HP 1 (Ortolani et al. 1997), NGC 6522 (Barbuy et al. 1994; Terndrup \& Walker 1994, Shara et al. 1998), NGC 6540 (Bica et al. 1994), NGC 6558 (Rich et al. 1998), NGC 6256 and NGC 6717 (Ortolani et al. 1999). On the other hand, the clusters Tonantzintla 2 (Bica et al. 1996), NGC 6380 and Terzan 12 (Ortolani et al. 1998), Terzan 3 and IC 1276 (Palomar 7) (Barbuy et al. 1998b), also located in the ring $5^{\circ}<r<20^{\circ}$ surrounding the central parts of the Galaxy, show a red horizontal branch (RHB) morphology. In the literature a number of clusters in the region have been studied by means of CCD, such as five RHB ones by Armandroff (1988).

We point out that most of the BHB clusters appear to have intermediate metallicities $(-1.5<[\mathrm{Fe} / \mathrm{H}]<-1.0)$, which we considered to form a family as discussed in Bica et al. (1998) and Rich et al. (1998). NGC 6517 with $[\mathrm{Fe} / \mathrm{H}]=-1.58$ also has a BHB (Kavelaars et al. 1995). NGC 6287 (Stetson \& West 1995), Terzan 4 (Ortolani et al. 1997a) and the present clusters Terzan 9, NGC 6139 and NGC 6453 seem to be in the very metal-poor class $([\mathrm{Fe} / \mathrm{H}] \approx-2.0)$. A fundamental question is which ones belong to the bulge or halo. In any case, spectroscopic studies of individual stars combined to proper motion determinations for all the blue HB sample are crucial to understand the early stages of the halo and bulge formation and evolution.

Acknowledgements. BB and EB acknowledge partial financial support from $\mathrm{CNPq}$ and Fapesp.

\section{References}

Armandroff T.E., 1988, AJ 96, 588

Armandroff T.E., 1989, AJ 97, 375
Armandroff T.E., Zinn R., 1988, AJ 96, 92

Barbuy B., Ortolani S., Bica E., 1994, 285, 871

Barbuy B., Bica E., Ortolani S., 1998a, A\&A 333, 17

Barbuy B., Ortolani S., Bica E., 1998b, A\&AS 132, 333

Bica E., Pastoriza M., 1983, ApSS 91, 79

Bica E., Alloin D., 1986, A\&A 162, 21

Bica E., Alloin D., 1987, A\&A 186, 49

Bica E., Ortolani S., Barbuy B., 1994, A\&A 283, 67

Bica E., Ortolani S., Barbuy B., 1996, A\&AS 120, 153

Bica E., Clariá J.J., Piatti A.E., Bonatto C., 1998, A\&AS 131, 483

Dean J.F., Warren P.R., Cousins A.J., 1978, MNRAS 183, 569

Guarnieri M.D., Ortolani S., Montegriffo P., Renzini A., Barbuy B., Bica E., Moneti A., 1998, A\&A 331, 70

Grebel A.K., Roberts W., 1995, A\&AS 109, 293

Harris W.E., 1996, AJ 112, 1487

Hazen-Liller M.L., 1984, AJ 89, 1551

Hazen M.L., 1991, AJ 101, 170

Kavelaars J.J., Hanes D.A., Bridges T.J., 1995, AJ 109, 2081

Landolt A.U., 1983, AJ 88, 439

Landolt A.U., 1992, AJ 104, 340

Larson R.B., 1990, PASP 102, 709

Liu T., McLean I., Becklin E., 1994, in Infrared Astronomy with Arrays, McLean I. (ed.). Kluwer Acad. Pub., p. 101

Malkan M.A., 1982, in Astrophysical Parameters for Globular Clusters, IAU Coll. 68, Philip A.G.D., Hayes D.S. (eds.). Schenectady: Davis, p. 533

Minniti D., 1995, AJ 109, 1663

Ortolani S., Bica E., Barbuy B., 1996, A\&A 306, 134

Ortolani S., Barbuy B., Bica E., 1997a, A\&A 319, 850

Ortolani S., Bica E., Barbuy B., 1997b, MNRAS 284, 692

Ortolani S., Bica E., Barbuy B., 1998, A\&AS 127, 471

Ortolani S., Barbuy B., Bica E., 1999, A\&AS 136, 237

Ortolani S., Renzini A., Gilmozzi R., Marconi G., Barbuy B., Bica E., Rich R.M., 1995, Nat 377, 701

Reid M., 1993, ARA\&A 31, 345

Reid M., 1998, AJ 115, 204

Rich R.M., Ortolani S., Bica E., Barbuy B., 1998, AJ 116, 1295

Rosino L., Ortolani S., Barbuy B., Bica E., 1997, MNRAS 289, 745

Samus N.N., Kravstov V.V., Pavlov M.V., Alcaino G., Liller W., Alvarado F., 1996, Astron. Lett. 22, 686

Shara M., Drissen L., Rich R.M., Paresce F., King I.R., Meylan G., 1998, ApJ 495, 796

Spergel D.N., Malhotra S., Blitz L., 1996, in Spiral Galaxies in the Near-IR, Minniti D., Rix H.-W. (eds.). Springer-Verlag, p. 128

Stetson P.B., West M.J., 1995, PASP 106, 726

Terndrup D.M., 1988, AJ 96, 884

Terndrup D.M., Walker A.R., 1994, AJ 107, 1786

Trager S.C., King I.R., Djorgovski S., 1995, AJ 109, 218

Webbink R.F., 1985, in Dynamics of Star Clusters, IAU Symp. 113, Goodman J., Hut P. (eds.). Dordrecht: Reidel, p. 541

Zinn R., 1980, ApJS 42, 19

Zinn R., 1985, ApJ 293, 424

Zinn R., West M.J., 1984, ApJS 55, 45

Zinn R., Barnes S., 1998, AJ 116, 1736 